Document downloaded from:

http://hdl.handle.net/10251/77093

This paper must be cited as:

Aguilar, A.; García Olcina, R.; Martos, J.; Soret, J.; Torres-Pais, J.; Benlloch Baviera, JM.; González Martínez, AJ.... (2014). Time of flight measurements based on FPGA using a breast dedicated PET. Journal of Instrumentation. 9:0-8. doi:10.1088/17480221/9/05/C05012.



The final publication is available at

http://dx.doi.org/10.1088/1748-0221/9/05/C05012

Copyright IOP Publishing

Additional Information 


\title{
Time of flight measurements based on FPGA using a breast dedicated PET
}

\author{
A.Aguilar ${ }^{\mathrm{a}}$, R.García Olcina ${ }^{\mathrm{a}}$, J.Martos ${ }^{\mathrm{a}}$, J.Soret ${ }^{\mathrm{a}}$, J.Torres ${ }^{\mathrm{a}}$, J.M.Benlloch ${ }^{\mathrm{b}}$, \\ A.J.González ${ }^{\mathrm{b}}$ and F.Sánchez \\ ${ }^{a}$ Communications and Digital Systems Design Group, University of Valencia \\ Av. De la Universidad, 46100, Burjassot, Spain \\ ${ }^{b}$ Institute for Instrumentation in Molecular Imaging (I3M). Centro Mixto UPV - CSIC - CIEMAT. \\ Camino de Vera s/n, 46022, Valencia, Spain \\ E-mail: albert.aguilareuv.es
}

ABSTRACT: In this work the implementation of a Time-to-Digital Converter (TDC) using a Nutt delay line FPGA-based and applied on a Positron Emission Tomography (PET) device is going to be presented in order to check the system's suitability for Time of Flight (TOF) measurements. In recent years, FPGAs have shown great advantages for precise time measurements in PET. The architecture employed for these measurements is described in detail. The system developed was tested on a dedicated breast PET prototype, composed of LYSO crystals and Positive Sensitive Photomultipliers (PSPMTs). Two distinct experiments were carried out for this purpose. In the first test, system linearity was evaluated in order to calibrate the time measurements, providing a linearity error of less than $2 \%$ and an average time resolution of $1.4 \mathrm{~ns}$ FWHM. The second set of measurements tested system resolution, resulting in a FWHM as good as $1.35 \mathrm{~ns}$. The results suggest that the coincidence window for the current PET can be reduced in order to minimize the random events and thus, achieve better image quality.

KEYWORDS: Time of Flight; Positron Emission Tomography; Field Programmable Gate Array; Position Sensitive Photomultipliers; Time-to-Digital Converter; 


\section{Contents}

1. Introducton $\quad 1$

2. System overview $r$

3. Characterization $r$

$\begin{array}{ll}3.1 \text { Time to Digital Converter } & 3\end{array}$

3.2 MAMMI breast prototype 4

4. Test and Results $\quad 5$

4.1 Test 1 . System calibration and linearity $\quad 5$

$\begin{array}{ll}4.2 \text { Test } 2 \text {. System resolution } & 6\end{array}$

$\begin{array}{ll}\text { 5. Summary and discussion } & 7\end{array}$

\section{Introduction}

Nuclear medicine has significantly improved in recent years due to the progress made in various technological fields such as electronics, mechanics and materials, to name but a few. Among them, Positron Emission Tomography (PET) systems have notably evolved thanks to the appearance of new types of sensors and technologies that improve scanner performance. One of the features that new PET systems incorporate is the ability to measure the Time of Flight (TOF) of the gamma particles arising from electron-positron annihilation. TOF determination allows locating the annihilation events within the line of response inside the system's field of view. When TOF is enabled, the algorithm of image reconstruction converges faster than in a system without this feature for a given number of detectors [1]. This has the advantage of application in scanners with certain detector apertures, as for instance those used in carrying out biopsy procedures, without reducing image quality.

There are several approaches for TOF determination. Traditionally, the use of custommanufactured circuits has been the most common. Such circuits, typically known as ASICs (Application Specific Integrated Circuits) need to be produced in high volumes to be costeffective in most cases. In addition to the fabrication issues, they are difficult to reconfigure. However, their design can be precisely adjusted in a way that significantly increases device performance.

More recently, new technologies offer the possibility of using devices with multiple capabilities at relatively low cost. Field Programmable Gate Arrays (FPGAs) came up as a possible alternative, both for their competitive cost and their re-programmability. Moreover, these devices have already been used for TOF calculations [2][3].

In this work, a set-up for TOF determination using a breast dedicated PET prototype is presented. The various parts of the set-up are explained, focusing on the TDC-FPGA implemented and providing the results of the first TOF measurements on the breast PET device. 


\section{System overview}

The experimental set-up used in this work is based on a dedicated breast PET and a custom trigger unit, as shown in Figure 1. The PET detector's outputs are connected to the PCB trigger unit in charge of determining the TOF of the gamma particles resulting from the positronelectron annihilation. The trigger is composed of Constant Fraction Discriminators (CFDs) and a FPGA, which contains a Time to Digital Converter (TDC) and an embedded software processor. A fast timing signal is distributed to different inputs of the trigger unit. Since the amplitudes of the signals (typically the last dynode of the PSPMTs, Position Sensitive Photomultiplier Tube) coming from the detectors are not always the same, the CFDs help to properly digitize the output signals [4]. If the amplitudes of the signal were constant, a leading edge could be used. Even so, alternatives such as double leading edge [5] must be undergoing to study in order to estimate the impact on the system performance.

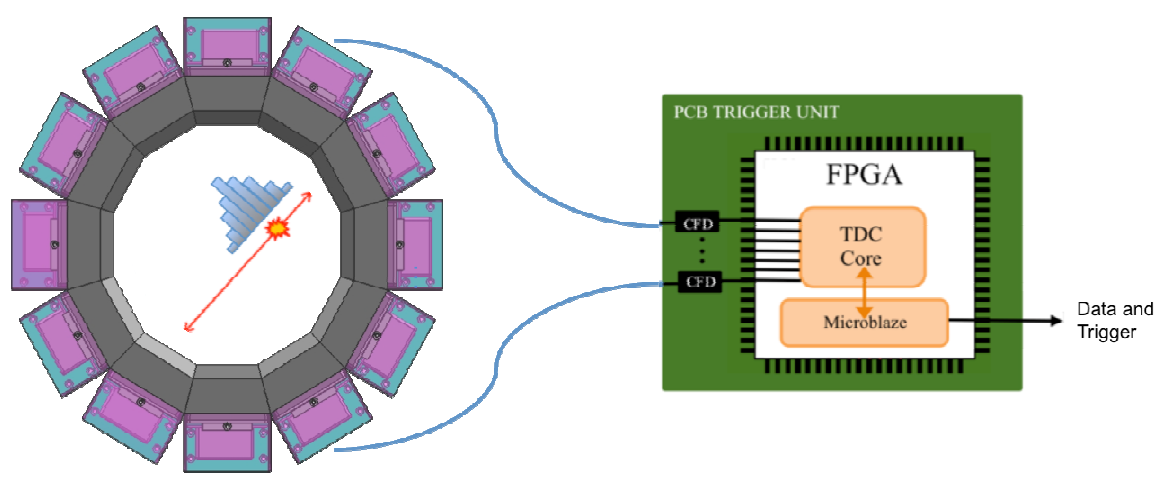

Figure 1. Architecture used for time measurements. The PCB trigger is composed of a digitizer for each detector module and an FPGA in charge of determining the TOF of the incoming particles.

The TDC generates a timestamp for each input signal, which is sent to the Microblaze, a Xilinx software processor core. They communicate with each other through Advanced Microcontroller Bus Architecture (AMBA) that is specially designed for 32-bit processors. Timestamps are stored in dedicated software registers and Microblaze checks if there is a true coincidence matching the timestamp differences of the confronted detectors. When this happens, it sends first the trigger signal to the acquisition PET system and then the value of the TOF. Once this data arrives, the acquisition system has at its disposal more information about the impact position inside the ring and it is easier for the software algorithm to reconstruct the image.

\section{Characterization}

The TDC architecture is based on a modified version of the Vernier structure called Nutt structure [6]. One part takes care of counting the system clock periods and the other of the subnanosecond measurements. The following subsections explain these two parts in more detail. 


\subsection{Time to Digital Converter}

The TDC is composed of a basic counter that counts the entire periods of the system clock and an accurate counter, which counts the intervals below the system clock period [7]. The first one is a classical counter that counts in increments of each system clock event. The second one is implemented using a delay line. The combination of both is known as the Nutt method and allows the counting of relatively high intervals of time with a high degree of precision.

There are multiple ways of implementing a delay line. One implementation could consist of two oscillators with different frequencies, which would start to operate with start/stop signals coming from outside the TDC. When the leading edges of the oscillators coincide, the measurement ends and, by knowing the period of the two oscillators, the original time interval can be deduced. The drawback of this method is the difficulty in implementing two oscillators with stable and small frequency differences.

There is another approach in which the two oscillators are replaced by two tapped delay lines. They are composed of a number of logic elements connected forming a chain for which the input signal is propagated (Figure 2, left side). The TDC implemented in this work relies on this last topology, using logic gates forming the delay line and registering their state through flip-flops [8]. One of the drawbacks of the structure adopted is that the delay lines need to be calibrated so that the delay of each tap is determined. An internal ring oscillator that is asynchronous to the system clock carries this out. It allows the sending of pseudo-random pulses to the delay line and counting the events registered by the flip-flop of each clock cycle. Since the delay line is configured in such a way that its total delay is higher than the system clock period, the more counts registered by a tap, the higher the delay associated with it. Therefore, it is possible to save the delay belonging to each tap and to count the number of taps reached for every incoming pulse in order to determine the timestamps. This calibration process is only performed once and when finished, the TDC is ready to start the measurements. The processing time depends on the number of pulses introduced but is normally less than $1 \mathrm{~ms}$.

Measurements of time differences were performed in order to check the TDC capabilities. Two channels were implemented and a signal coming from a function generator was split into two branches and each of them injected into the TDC. Sub-nanosecond time resolution was tested following the scheme depicted in Figure 2, right side. The results have been Gaussian adjusted and a standard deviation of 31.8 ps has been achieved for a pair of channels, resulting in a FWHM of $75 \pm 5$ ps.
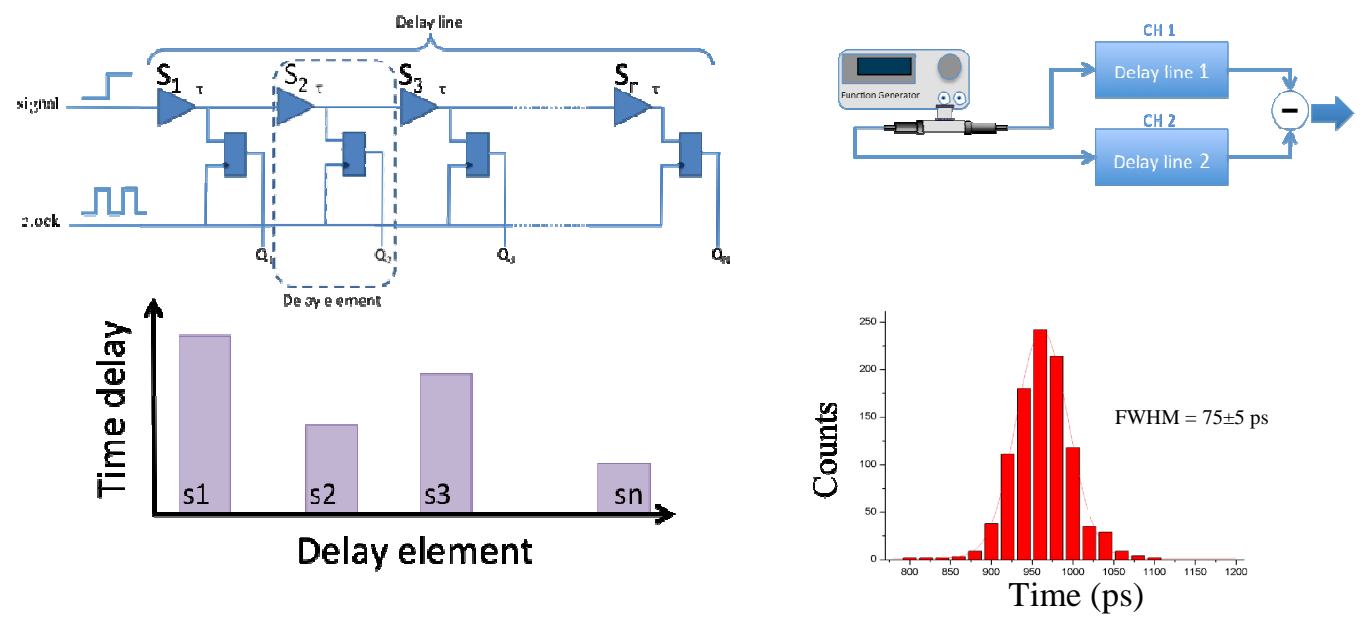

Figure 2. Adopted delay line structure based on the individual delay gate determination (left). Scheme of time difference measurements and histogram of the acquired values (right). 


\subsection{MAMMI breast PET prototype}

MAMMI is a dedicated breast PET suitable for the detection of small size tumor lesions as well as for cancer therapy assessment [9][10]. MAMMI consists of 12 identical detector modules with a scanner aperture of $186 \mathrm{~mm}$. Each detector is composed of a single black painted monolithic Cerium-doped Lutetium Yttrium Orthosilicate (LYSO) crystal measuring 50x50 $\mathrm{mm}^{2}$, a PSPMT (Hamamatsu, model H8500) and an electronics board composed of a high voltage power supply and conditioning circuits. LYSO is suitable for PET systems due to its high light yield, fast response time and high density. The pyramidal shape of the crystals is used to minimize dead areas and increases the overall sensitivity of the scanner.

The last dynode signal of the PSPMT is conditioned by an operational amplifier, which amplifies and inverts the signal that will be used for timing measurements, the shape of which is shown in Figure 3 right.


Figure 3. From left to right: MAMMI breast prototype; a close-up of the MAMMI ring; the output signal of the last dynode of a sensor after conditioning through an operational amplifier.

An energy spectrum is presented in Fig. 4. It corresponds to one of the modules of the ring and for a centered region of interest of roughly $5 \times 5 \mathrm{~mm}^{2}$. An energy resolution of $\approx 15 \%$ can be obtained from this plot. It also depicts the ADC linearity with a ratio of the gamma line at $1274 \mathrm{keV}$ to the $511 \mathrm{keV}$ photopeak energy of 2.48 measured (2.49 theoretical).



Figure 4. Energy spectrum corresponding to a single module. 


\section{Test and Results}

\subsection{System calibration and linearity}

In this first test, a radioactive ${ }^{22} \mathrm{Na}$ source of $1 \mathrm{~mm}^{3}$ active area was placed on the front face of one detector whereas that used for the coincidence was located at different positions with incremental distances of $5 \mathrm{~cm}$, as the next figure shows. The two last dynode outputs of both detectors were directly injected into the CFDs, taking into account the rise time $\left(t_{r}\right)$ of the signals to configure the delay time of the device. The CFD generates a bipolar output signal, which is the sum of two copies of itself: one delayed and inverted and the other one attenuated. Using such a method and with the proper configuration of its parameters, it is possible to achieve simultaneous zero crossing of all signals and in doing so, minimize the time walk. As has been briefly mentioned in section 2, the time walk is a time shift due to the amplitude differences between the sensor output signals, being that the rise time is independent of the signal amplitude. If the pulse rise time is defined as the time from $10 \%$ to $90 \%$ of the signal maximum, the delay must be equal to or less than (50\%-80\%) this rise time in order to minimize the walk time. The internal delay of the CFD must also be considered in establishing the final delay time which, depending on the design, may vary by a few nanoseconds. In the present configuration, the rise time of the signal was measured at about $6 \mathrm{~ns}$ and, according to the principles mentioned above, the delay has been fixed at $5 \mathrm{~ns}$.

A dark blanket was used to cover both modules and protect them from the ambient light. The measurements were carried out for about 2,000 samples. The data were fitted to a Gaussian distribution, recording both its centroid and the standard deviation.

Figure 5 right depicts the centroid of each distribution as a function of the detectors distance. In the $\mathrm{Y}$ axis is represented the expected time steps of the six points measured, whereas in the $\mathrm{X}$ axis is shown the time differences measured. The determined regression coefficient resulted in 0.9985 . Figure 6 shows the six distributions measured. The average Full Width at Half of the Maximum (FWHM) was found to be $1.416 \mathrm{~ns}$ with a small deviation of only $30 \mathrm{ps}$, which is an error of less than $2 \%$. This experiment shows the high linearity of our measuring procedure and, moreover, provides us with an accurate time calibration method.
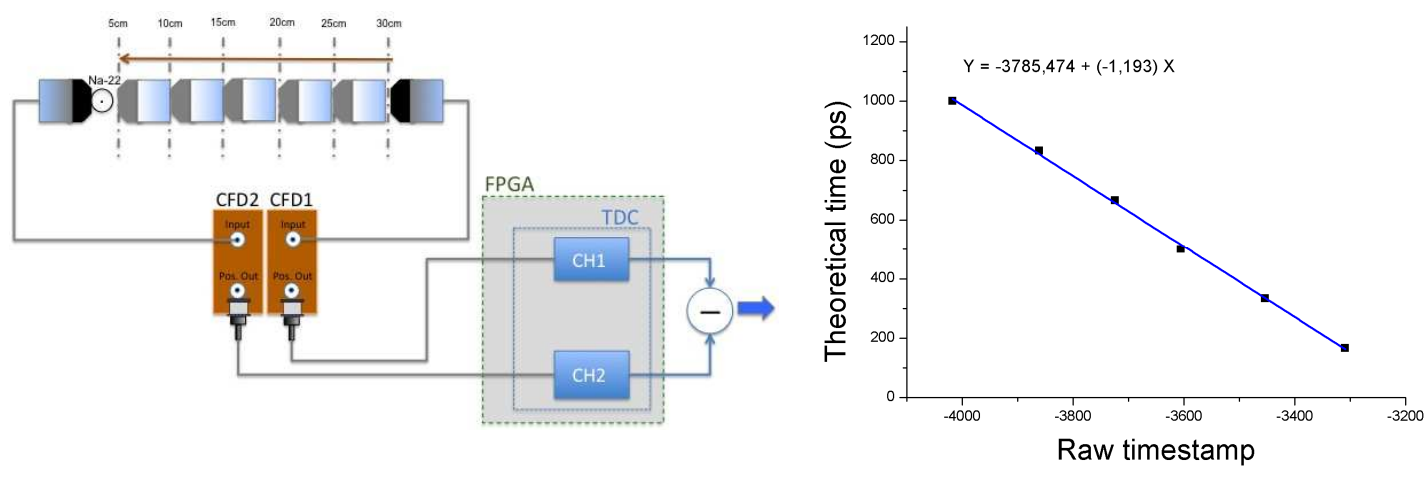

Figure 5. Left, linearity set-up. Right, results of the measured centroids. 


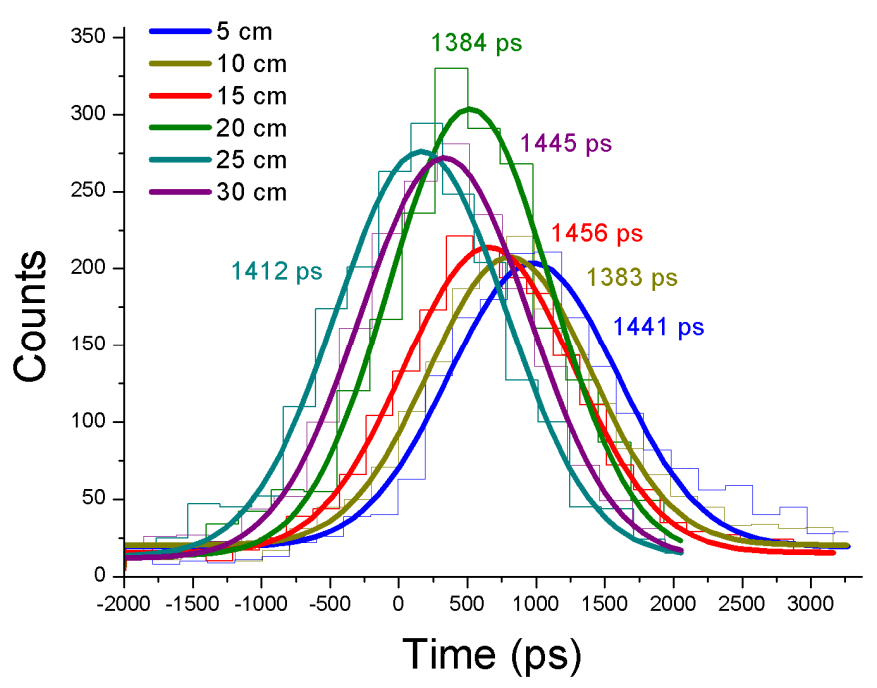

Figure 6. Representation of the Gaussian distributions referred to Figure 5.

\subsection{System resolution}

The electronics designed for TOF capabilities were tested with two opposite detectors of the MAMMI system recording the arrival time of coincidence events. The ${ }^{22} \mathrm{Na}$ source was placed at three different radial positions in the scanner field of view, namely the center and the edges of the PET aperture. The same conditions were applied for both the detectors and the CFDs as in the prior experiment. The time difference was measured, providing information on the source location within the ring, as shown in Figure 6 right. The profiles were fitted to a Gaussian distribution and their centroids and FWHM recorded. The measurements resulted in Gaussian centroids of $-612,-9.3$ and $640 \mathrm{ps}$ (from left to right). Therefore, the distance between the twoborder positions with respect to that in the center are 602.75 and $649.29 \mathrm{ps}$, i.e., the distance between centroids is $1,252.05$ ps. The Gaussian fits resulted in a centroid error of about 15 ps.

FWHM values are all about $1.4 \mathrm{ns,}$ as expected according to the results of the previous experiment.

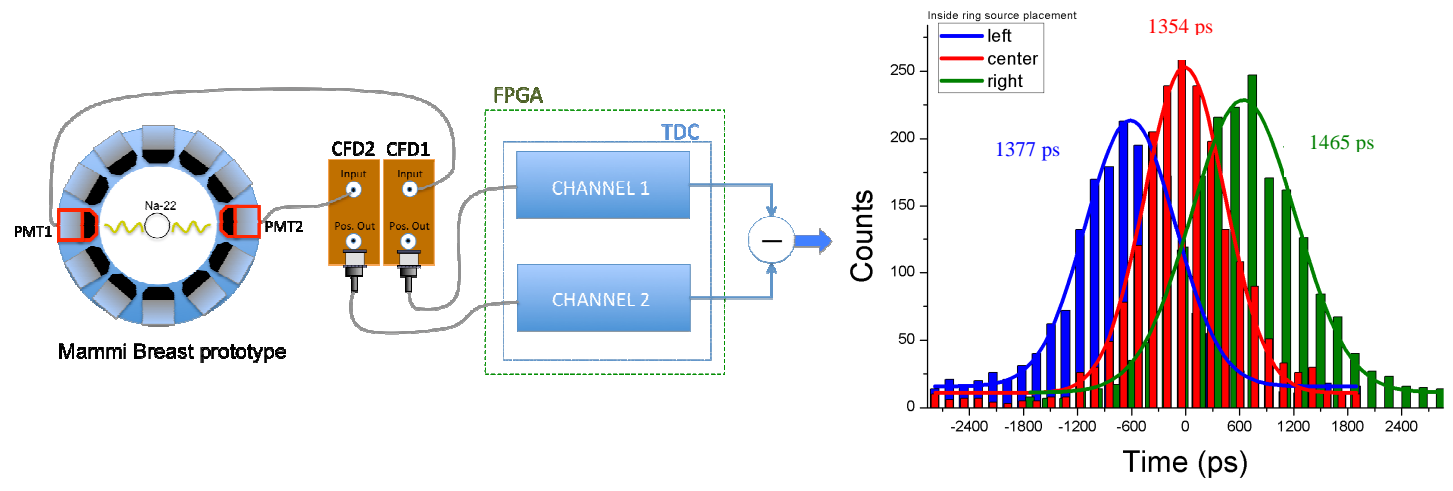

Figure 7. Timing measurement set-up used to test the system capability to estimate the TOF of the gamma particles produced by the radioactive source. 
The distance between opposing front crystal faces is $190 \mathrm{~mm}$. Since the most probable depth of interaction point is about $4.2 \mathrm{~mm}$ from this surface [11], there is a maximum TOF distance of about 661 ps. Therefore, the expected distance between the centroid of the TOF distributions of sources located on opposite edges of the ring should be of 1,326 ps.

There is a difference of $100 \mathrm{ps}$ between the theoretical value (1,326 ps) and the obtained value (1,252 ps). The reason for the disparity in times could be due to an error in the positioning of the source, which is assumed to be about $1 \mathrm{~cm}( \pm 33 \mathrm{ps})$. Moreover, the error in the Gaussian fit adjust function can be considered to be about 15 ps. Thus, the spatial positioning and centroid uncertainties obtained during the experiment are estimated to be 66 and $30 \mathrm{ps,}$ respectively. Summarizing, the experimental value with the error bars 1,252 $\pm 66 \pm 30$ ps is in agreement with the theoretical value of $1,326 \mathrm{ps}$ (without including uncertainties in the depth of interaction estimation).

\section{Summary and discussion}

The TDC developed has been tested with a breast PET prototype for TOF measurements. A setup has been implemented to enable TOF measurements to be carried out. The proposed tests have demonstrated the viability of accurately measuring times that will result on a system performance improvement. .

In the resolution test, the system capability of distinguishing the position of the source in few locations inside the ring has been shown. Due to the high transit time spread of the PSPMTs used (about 400 ps nominal) and electronic jitter, the best resolution achieved for this system has been limited to about $1 \pm 0.03 \mathrm{~ns}$ FWHM. It seems that the conditioning electronic, which returns the signal shape shown in Fig. 3, is not responsible of the time resolution deterioration since the signal rise time remains constant after its conditioning. With the proper data treatment, it might be possible to distinguish 3 regions inside the scanner field of view.

During the system linearity test the registration of coincidences in six positions was demonstrated, resulting in a regression coefficient of 0.99 . Furthermore, the total deviation of the FWHM of the Gaussian distributions for the six cases has been 74 ps for a resolution of 1.4 ns on average. In Figure 7 it can be appreciated a 15 counts offset for all distributions. This continuous distribution is most likely due to random coincidences, although other contributions such as scatter events and the natural radiation of the Lutetium contained in the LYSO crystals could also contribute.

The obtained results are in agreement with the literature. For example, in [12], the authors also used the H8500 PSPMTs coupled to LYSO crystals $\left(20 \times 20\right.$ array of $\left.2 \times 2 \times 10 \mathrm{~mm}^{3}\right)$, being these pixelated instead of monolithic. They use a commercial data acquisition system for collecting and manage the data using a coincidence window of $12 \mathrm{~ns}$. With this configuration, they obtain a $1.16 \mathrm{~ns}$ resolving time. But it has to take into account that pixelated crystals offers a better performance compared to monolithic due to the higher scintillation light transmitted to the photosensor, which is a crucial factor to improve the system performance.

The time resolution achieved with the TDC and the PET system could reduce the coincidence window to a value of between 1.5 and $2 \mathrm{~ns}$ (in this prototype the coincidence window was set to $5 \mathrm{~ns}$ in most of the experiments $[9,10])$. This will decrease the number of acquired random events, which for a breast dedicated PET might be significant. Certainly, this fact would translate into improvements in the reconstructed image. 
Finally, the results suggest that by improving the discrimination electronics of the dedicated breast PET, it might be possible to reach higher detector performance, namely spatial resolution.

\section{References}

[1] M. Conti, State of the art and challenges of time-of-flight PET, Physics in Medicine and Biology, 25, 1-11 (2009).

[2] W. Gao, et al., Integrated High-Resolution Multi-Channel Time-to-Digital Converters (TDCs) for PET Imaging, N. Laskovski (Ed.), ISBN: 978-953-307-475-7.

[3] M. Haselman et al., FPGA-Based Front-End Electronics for Position Emission Tomography, NIHPA, 93-102 (2009)

[4] J. Liu et al., Monte Carlo Simulation Study on the Time Resolution of a PMT-Quadrant-Sharing LSO Detector Block for Time-of-Flight PET, IEEE NSS Conference Record M18-2, 3294-3304 (2007).

[5] A. Gola, C. Piamonte, A. Tarolli, The DLED algorithm for timing measurements on large area SiPMs Coupled to Scintillators, IEEE Transactions on Nuclear Science, Vol. 59, No. 2, 358-365 (2012).

[6] J. Kalisz, M. Pawlowski, R. Pelka, Error analysis and design of the Nutt $\square$ time-interval digitiser with picosecond resolution, J. Phys. E: Sci. Instrum. 20: 1330-1341 (1987).

[7] J. Torres et al., High resolution Time of Flight determination based on reconfigurable logic devices for future PET/MR systems, Nuclear $\square$ Instruments \& Methods In Physics Research A, 702 pp. 73 76 (2013).

[8] J. Torres, et al., Time-to-digital converter based on FPGA with multiple channel capability, Transactions on Nuclear Science, ISSN: 0018-9499, Issue: 99 (2013)

http://ieeexplore.ieee.org/xpl/articleDetails.jsp?arnumber=6661408

[9] L. Moliner et al., Design and evaluation of the MAMMI dedicated breast PET, Med. Phys. Vol. 39, No.9, pp. 5393-5404 (2012).

[10] A. González et al., Design and first results of an innovative and dedicated breast PET, Current Medical Imaging Reviews, Vol.8, pp. 144-150 (2012).

[11] A. Soriano et al., Minimization of Parallax Error in Dedicated Breast PET, IEEE Transactions on Nuclear Science, Vol. 60, No.2, pp. $739-745$ (2013).

[12] H. Alva-Sánchez et al., Initial characterization of benchtop microPET system based on LYSO crystal arrays and Hamamatsu H8500 PS-PMTs, Nuclear $\square$ Instruments \& Methods In Physics Research A, 604 pp. 335-338 (2009). 